Wichtigsten ist und was gegebenenfalls wegfallen könnte. Hier Tipps zur Vorbereitung:

_ Sammeln Sie in den Monaten vor der Auszeit alle möglichen Ideen (bei mir war das ein großer DIN-A4-Bogen am Kühlschrank, auf dem schließlich 30 Einzelideen standen).

- Machen Sie sich dann klar, was Sie mit der Sabbatzeit primär wollen. Ein oder zwei konkret gewählte Ziele sind meist besser als zu allgemeine wie „Abenteuer erleben“ oder „mich verändern“.

_Überlegen Sie, wie flexibel Sie sein wollen. Ein zu enges Raster verhindert vielleicht genau die Erfahrungen, die Sie suchen.

-Wenn etwas ganz und gar nicht klappt, verändern sie Ihre Ziele räumlich, zeitlich et cetera.

_Bei einer längeren Auszeit rechnen Sie mit Phasen der Langeweile.

_Tiefere Einsichten, persönliche Veränderungen, Umorientierung individueller Werte sind bei einer längere Auszeit eher die Regel als die Ausnahme.

_Überlegen Sie vorher gut, ob Sie die Auszeit allein oder zu zweit verbringen wollen. Zu zweit ist man weniger allein, unbewusst bremst man aber womöglich Ansätze tieferer Veränderungen aus.

_Planen Sie eine Wiedereinstiegsphase ein! Speziell nach vielmonatigem Aufenthalt im Ausland kann die Rückkehr nach Deutschland einen unerwarteten Kulturschock mit sich bringen. Lassen Sie sich zwei Wochen Zeit, um wieder anzukommen.

_Wenn möglich, gönnen Sie sich vor der Sabbatzeit eine professionelle Beratung. Das könnte Sie auf den einen oder anderen blinden Fleck aufmerksam machen und wichtige Unterstützung in Ihrem Vorhaben geben.

Fazit

1. Ein Sabbatical kann „lebensstiftende Erfahrungen" für einen Arzt bedeuten, es kann ihm die Möglichkeit bieten, über den weiteren Berufsweg nachzudenken, neue berufsbezogene Fähigkeiten zu erwerben, neue Begeisterung für die ärztliche Tätigkeit zu finden.

2. Die zögerliche Haltung vieler Arbeitgeber, Ärzten eine Auszeit zuzugestehen, lässt sich als kurzsichtig und beschämend qualifizieren. Sie riskiert unnötigerweise, dass wir noch mehr Kollegen aus dem aktiven Beruf verlieren durch Burn-out, Depression, Sucht, Suizid und vieles mehr!

3. Für den einzelnen Arzt kann ein Sabbatical ein sinnvoller Coping-Mechanismus sein bei einem Übermaß an Stress und Erschöpfung. Ärzte, die eine Sabbatzeit genommen haben, sehen diese fast immer als extrem wertvolle Erfahrung für den Beruf und das Privatleben an. Insofern ist der zeitlich limitierte Luxus langfristig auch positiv für die Patientenversorgung.

\section{Sabbatical \\ Egoistischer Akt oder berufliche Notwendigkeit?}

Wir Ärzte sind in der Regel sehr pflichtbewusst, belastbar und motiviert durch ein hohes Berufsethos. Die meisten von uns finden große Befriedigung in der Versorgung der Patienten. Zugleich trainiert uns jedoch die medizinische Sozialisation dazu, dieses Ideal im Sinne einer Selbstausbeutung immer und überall an die erste Stelle zu setzen.

Vielen Kollegen tut dies auf die Dauer eines langen Berufslebens eindeutig nicht gut. Es kommt zu Übermüdung, Selbstentfremdung, Zynismus, Suchtgefährdung, Sinnverlust, auch innerpartnerschaftliche Entfremdung („Du bist nie da, wenn ich Dich brauche.") und stressbedingten Krankheiten. In meiner Praxis behandle ich viele Kollegen mit diesen Störungen und frage mich des Öfteren, ob wir Ärzte denn nicht auch ein Recht auf Erhalt und Pflege unserer Gesundheit haben. Müssen wir uns in der Praxis kaputt machen?

Hier sehe ich die Chance einer Auszeit. Auf lange Sicht nützt den Patienten ein gesunder, zufriedener Arzt mehr als ein überarbeiteter, dauergestresster. Es ist sinnvoll, auch über die Art unserer Tätigkeit zu reflektieren. Warum sind wir seinerzeit in die Medizin gegangen, wie könnten wir authentischer in unserem Tun sein? Die Berichte von Kollegen nach einer Auszeit zeigen größere Ausgewogenheit, mehr Abstand, deutlich mehr Entschlossenheit nach Wegen zu besserer Work-Life-Balance zu suchen.

Dass letztlich auch die Patienten von einem Sabbatical ihres Arztes profitieren, erklärte eine US-amerikanische Fachärztin so: „Und was passiert mit meinen Patienten, die ich im Stich gelassen habe? Nun, alles Gute kommt irgendwann zurück; auf Dauer werden sie besser behandelt, wenn wir wieder lieben können, was wir tun."

4. Unabdingbar ist eine gründliche Vorplanung, welche die arbeitsrechtliche/vertragliche Situation, die finanziellen Möglichkeiten, die Anwerbung von Vertretern und auch die inhaltlichen Ziele des Sabbatical umfassend klärt. Hierbei sind eine professionelle Begleitung und auch der Austausch mit sabbaterfahrenen Kollegen sehr zu empfehlen.

Dr. med. Bernhard Mäulen Institut für Ärztegesundheit Vöhrenbacherstraße 4

78050 Villingen 